\title{
Higher order model power calculation of the 56 MHz SRF cavity
}

\author{
E.M. Choi
}

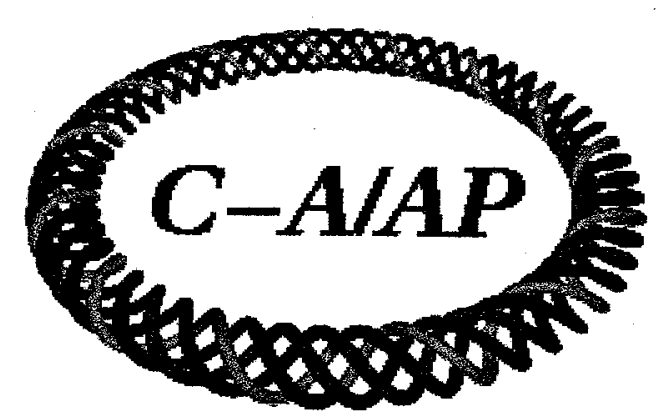

\section{Collider-Accelerator Department Brookhaven National Laboratory Upton, NY 11973}

Notice: This document has been authorized by employees of Brookhaven Science Associates, LLC under Contract No. DE-AC02-98CH10886 with the U.S. Department of Energy. The United States Government retains a nonexclusive, paid-up, irrevocable, world-wide license to publish or reproduce the published form of this document, or allow others to do so, for United States Government purposes. 


\title{
Higher order mode power calculation of the $56 \mathrm{MHz}$ SRF cavity
}

\author{
E. M. Choi
}

August 28, 2008

\begin{abstract}
In this report, the HOM power dissipated to the load in the $56 \mathrm{MHz}$ RF cavity is calculated. The HOM frequencies and $\mathrm{Q}$ factors with the inserted HOM damper are obtained from the simulations by MWS and SLAC codes.
\end{abstract}

\section{Introduction}

A $56 \mathrm{MHz}$ superconducting RF cavity (SRF) is going to be installed in Relativistic Heavy Ion Collider (RHIC) ring for the luminosity upgrade of the RHIC operation. Currently, it is being designed and prototype-tested as an Accelerator Improvement Project (AIP). The $56 \mathrm{MHz}$ SRF cavity will be turned on at store of the RHIC operation. When the beam frequencies overlap or come close to the cavity HOM frequencies, the HOMs are excited in the cavity, which may lead to beam instability. Since the shunt impedance of the superconducting cavity tends to be higher than that of the copper cavity, the voltage developed in the cavity due to the HOM excitation can in principle be large. Therefore, supressing the HOMs is an important task for the $56 \mathrm{MHz}$ SRF cavity. To pursue the task, understanding the frequencies, Q factors and shunt impedances for the HOMs is prerequisite. Based on the given HOM characteristic, the HOM power can be calculated. The simulation results from the available programs as well as the prototype measurement results are used for the analysis.

\section{The HOM damper simulation}

The HOM damper location has been chosen to be inserted through the chemical port openings as shown in Fig. 1. It allows the cavity engineering design to be simpler. The HOM damper size is limited by the chemical port opening size. A radius of $1.74 \mathrm{~cm}$ of chemical port opening allows a square type of HOM damper loop to be $6 \mathrm{~cm}$ by $2.88 \mathrm{~cm}$ with $2 \mathrm{~cm}$ width.

The two codes (Microwave Studio (MWS) [1] and Omega3P SLAC code [2] are used for the simulations. The results will be discussed and the comparison between the MWS and Omega3P is included. Fig. 1 is the MWS model for the $56 \mathrm{MHz}$ with the HOM damper loop. The eigenmode solver was used for obtaining the cavity HOM frequencies, shunt impedances, and external Q factors. Symmetry is applied for both horizontal (xz plane) 


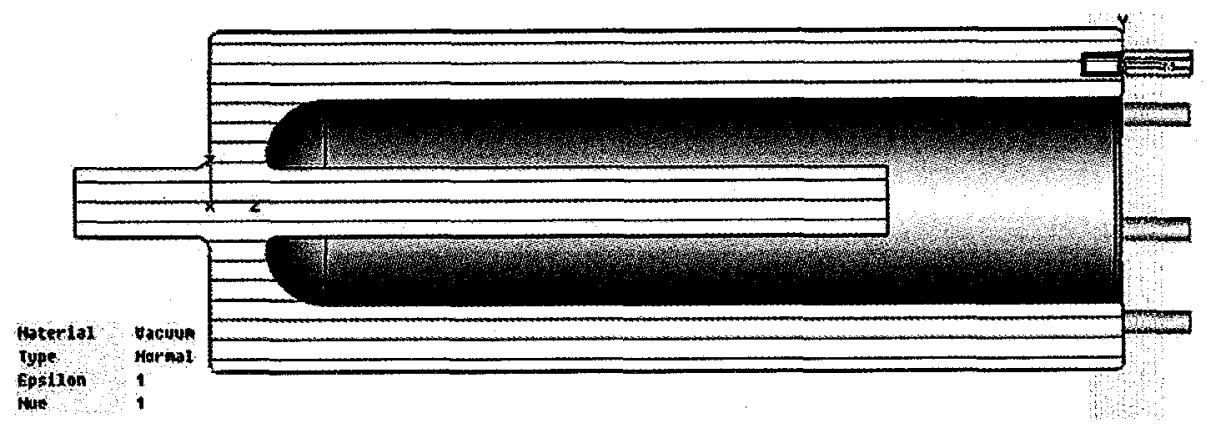

Figure 1: A MWS model of the $56 \mathrm{MHz}$ cavity with a HOM damper inserted through the chemical cleaning port opening

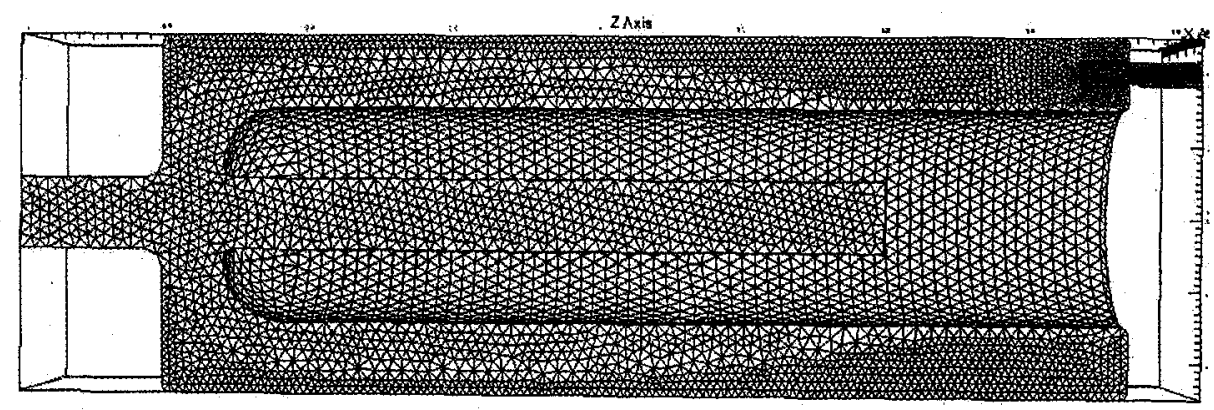

Figure 2: An Omega3P cavity model of the $56 \mathrm{MHz}$ cavity with an inserted HOM damper filled with meshcells

and vertical (yz plane) planes, which reduces the time for simulation. The frequency for simulation was spanned from $50 \mathrm{MHz}$ up to $1.2 \mathrm{GHz}$, which was broken to small frequency spans for reducing the number of the meshcells (MWS adapts the mesh size based on the frequency).

Recently, Omega3P code developed by Kwok Ko at al. in SLAC becomes available in the SRF and ecooling group in BNL. The code has been benchmarked against experiments and other commercial/educational codes in various laboratories. The Omega3P code is an eigenmode solver that runs massively in the parallel computer which makes a complicated geometry run much faster than other codes. Currently, the code is running in Bassi system that is a distributed memory computer with 888 processors in National Energy Research Scientific Computing Center (NERSC) account.

Fig. 2 shows the $56 \mathrm{MHz}$ cavity model in Omega3P which is filled with 493850 meshcells. The cavity geometry and the HOM damper is identical to the MWS model. A half of the full cavity geometry was simulated as shown in Fig. 2. The geometry and mesh generation was done by Cubit program [3]. After the mesh is generated by Cubit, it is converted and interpreted in SLAC code using "acdtool" code. The Omega3p is running with the prepared mesh file. Fig. 3 shows the generated E field profiles by Omega3p 


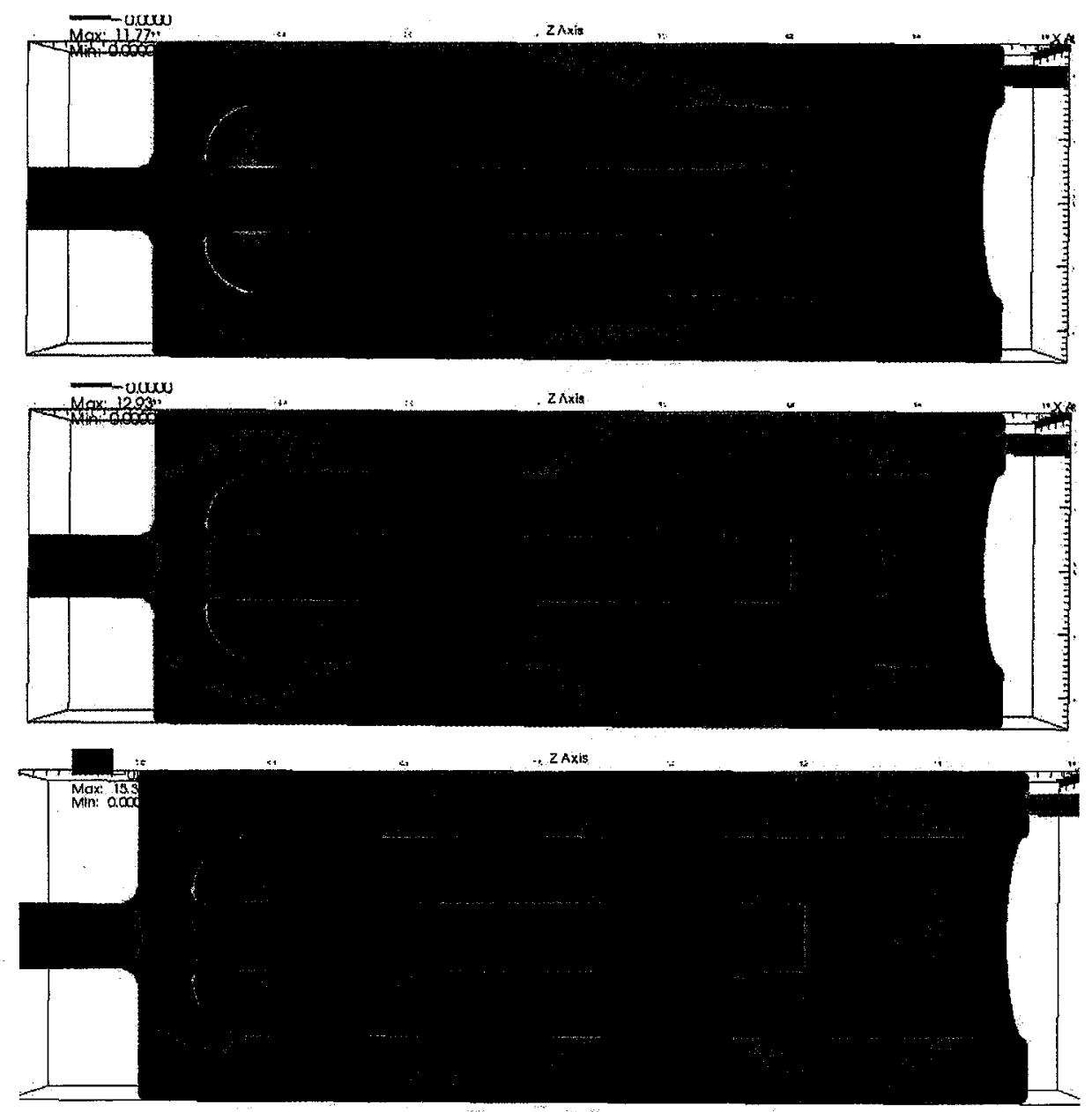

Figure 3: Electric field profiles from the Omega3p simulation; $56.263 \mathrm{MHz}$ (top), $167.865 \mathrm{MHz}$ (middle), and $276.205 \mathrm{MHz}$ (bottom). 
Table 1: Calculated monopole frequencies, external $\mathrm{Q}$ factors and $\mathrm{R} / \mathrm{Q}$ values from the Omega3P and the MWS in the $56 \mathrm{MHz}$ cavity loaded with the HOM damper loop

\begin{tabular}{cc|cc|cc}
\hline $\begin{array}{c}\text { Omega3P } \\
\text { Frequency (MHz) }\end{array}$ & $\begin{array}{c}\text { MWS } \\
\text { Frequency (MHz) }\end{array}$ & $\begin{array}{c}\text { Omega3P } \\
Q_{\text {ext }}\end{array}$ & $\begin{array}{c}\text { MWS } \\
\text { Qext }\end{array}$ & $\begin{array}{c}\text { Omega3P } \\
\text { R/Q }\end{array}$ & $\begin{array}{c}\text { MWS } \\
\text { R/Q }\end{array}$ \\
\hline 56.263 & 56.241 & 3485 & 4255 & 80.0 & 78.9 \\
167.865 & 167.790 & 1694 & 1818 & 32.3 & 30 \\
276.205 & 276.147 & 1704 & 1614 & 27.5 & 23.2 \\
378.447 & 378.393 & 2073 & 1780 & 27.9 & 22.6 \\
475.459 & 475.393 & 2520 & 2040 & 21.7 & 21.4 \\
574.779 & 574.724 & 3127 & 2580 & 13 & 15.8 \\
680.133 & 680.038 & 2802 & 2520 & 6.7 & 9.8 \\
789.259 & 789.042 & 4108 & 3800 & 3.5 & 6.2 \\
899.415 & 899.121 & 37142 & 50400 & 2.8 & 4.9 \\
1009.348 & 1008.668 & 4577 & 5726 & 2.8 & 6.5 \\
1112.723 & 1112.011 & 5401 & 4900 & 14.9 & 23.4 \\
1138.591 & 1137.910 & 10663 & 11060 & 17.7 & 11.6 \\
\hline
\end{tabular}

simulation at the first three monopole modes. The HOM damper is located at the end of the cavity, which is the region of the strongest magnetic field. Table 1 is a summary of the HOM frequencies, $\mathrm{Q}$ factors, and $\mathrm{R} / \mathrm{Q}$ values done by the Omega3P and the MWS. More detailed information about the HOMs including the mode configurations can be found in [5]. The comparison between the Omega3P and the MWS simulations shows that the frequency, $\mathrm{Q}$ factor and $\mathrm{R} / \mathrm{Q}$ results are in reasonable agreement.

\section{The analytic expression of the HOM power}

From the obtained HOM frequencies, external $\mathrm{Q}$ factors, and R/Q values from the simulations summarized in Table 1, the HOM power dissipated to the load can be calculated. From the recent study on the $28 \mathrm{MHz}$ HOM power measurement [6], it was shown that the HOM power calculation agrees very well to the measured HOM power. Therefore, the same analysis can be done to estimate the HOM power for the $56 \mathrm{MHz}$ cavity case. First of all, the set up for the HOM calculation is reviewed. The beam current with an infinitely long train of point-charge bunches can be expressed in a time domain as

$$
I(t)=\sum_{k=-\infty}^{\infty} \sum_{i=1}^{N_{1}} q_{i} \delta\left(t-k T_{0}-\frac{i T_{0}}{N_{1}}\right)
$$

where $q_{i}$ is the $\mathrm{i}$-th bunch charge. $N_{1}$ is the number of buckets and $T_{0}$ is the revolution period. The beam current with an infinitely long train of gaussian bunches, which is much closer to the real situation is as follows.

$$
I(t)=\sum_{k=-\infty}^{\infty} \sum_{i=1}^{N_{1}} \frac{q_{h}}{\sqrt{2 \pi \sigma_{t}^{2}}} \exp \left[-\frac{\left(t-k T_{0}-h T_{0} / N_{1}\right)^{2}}{2 \sigma_{t}^{2}}\right] .
$$


Table 2: The beam parameter for the proton run

\begin{tabular}{c|c}
\hline Bunch intensity & $2 \times 1.6 \times 10^{11}$ protons \\
Number of bunches $\left(\mathrm{N}_{1}\right)$ & 120 \\
Rms bunch length $\left(\sigma_{t}\right)$ & $2.4 \mathrm{~ns}$ \\
\hline
\end{tabular}

The Fourier transfered beam current of Eq. 2 will be used for the calculation, therefore, the beam current in frequency domain is given as follows.

$$
I_{n}=\frac{1}{T_{0}} \int_{0}^{T_{0}} I_{0}(t) \exp \left(j \frac{2 \pi n}{T_{0}} t\right) d t \cong \sum_{h=1}^{N_{1}} \frac{q_{h}}{T_{0}} \exp \left(j \frac{2 \pi n h}{T_{0}}\right) \exp \left[-\left(\frac{2 \pi n}{T_{0}}\right)^{2} \frac{\sigma_{t}^{2}}{2}\right] .
$$

The real part of the impedance of the cavity is given as follws [7],

$$
\operatorname{Re}[Z(\omega)]=\frac{(R / Q)_{k} Q_{k}}{1+Q_{k}^{2}\left(\frac{\omega}{\omega_{r}}-\frac{\omega_{r}}{\omega}\right)^{2}}
$$

where $k$ is the $\mathrm{k}$-th mode of the cavity, $\mathrm{Q}_{k}$ is the loaded $\mathrm{Q}$ factor of the $\mathrm{k}$-th mode, and $\omega_{r}$ is the beam revolution frequency. Therefore, the power released by the beam to the $\mathrm{k}$-th cavity mode can be expressed as follows.

$$
P_{k}=\sum_{n=0}^{\infty} \frac{2(R / Q)_{k} Q_{k} I_{n}^{2}}{1+Q_{k}^{2}\left(\frac{\omega}{\omega_{r}}-\frac{\omega_{r}}{\omega}\right)^{2}}
$$

where $n$ is the harmonic number of the beam.

\section{The calculation of the HOM power}

For estimating the HOM power of the $56 \mathrm{MHz}$ cavity with the HOM damper, the case which gives the highest HOM power is considered, which is the proton run case. For the proton run, the bunch intensity is around $1.6 \times 10^{11}$ protons, and the number of bunches is 120 . Since the $56 \mathrm{MHz}$ cavity is going to be installed in the common area of the RHIC rings, the twice higher beam current should be used in the calculation. It turns out from the $28 \mathrm{MHz}$ cavity study [6] that the bunch length is the most important and critical parameter for the proper HOM power calculation. For the $56 \mathrm{MHz}$ cavity, the bunch length of 2.4 ns should be used in the calculation, which is smaller than that of the $28 \mathrm{MHz}$ cavity. Table 2 summarizes the key values for the proton run with the $56 \mathrm{MHz}$ cavity on.

Fig. 4 is the plots of the frequency content of the beam. As seen in the bottom plot of Fig. 4 , the gaussian bunch beam, which may represent the real case, has a strong decay of the beam current as the frequency increases, which will affect significantly the HOM power at high HOM frequencies. In order to calculate the HOM power correctly, the $56 \mathrm{MHz}$ cavity run scenario has to be carefully considered. At injection and acceleration, the fundamental mode of the $56 \mathrm{MHz}$ cavity will be damped so that the beam does not 

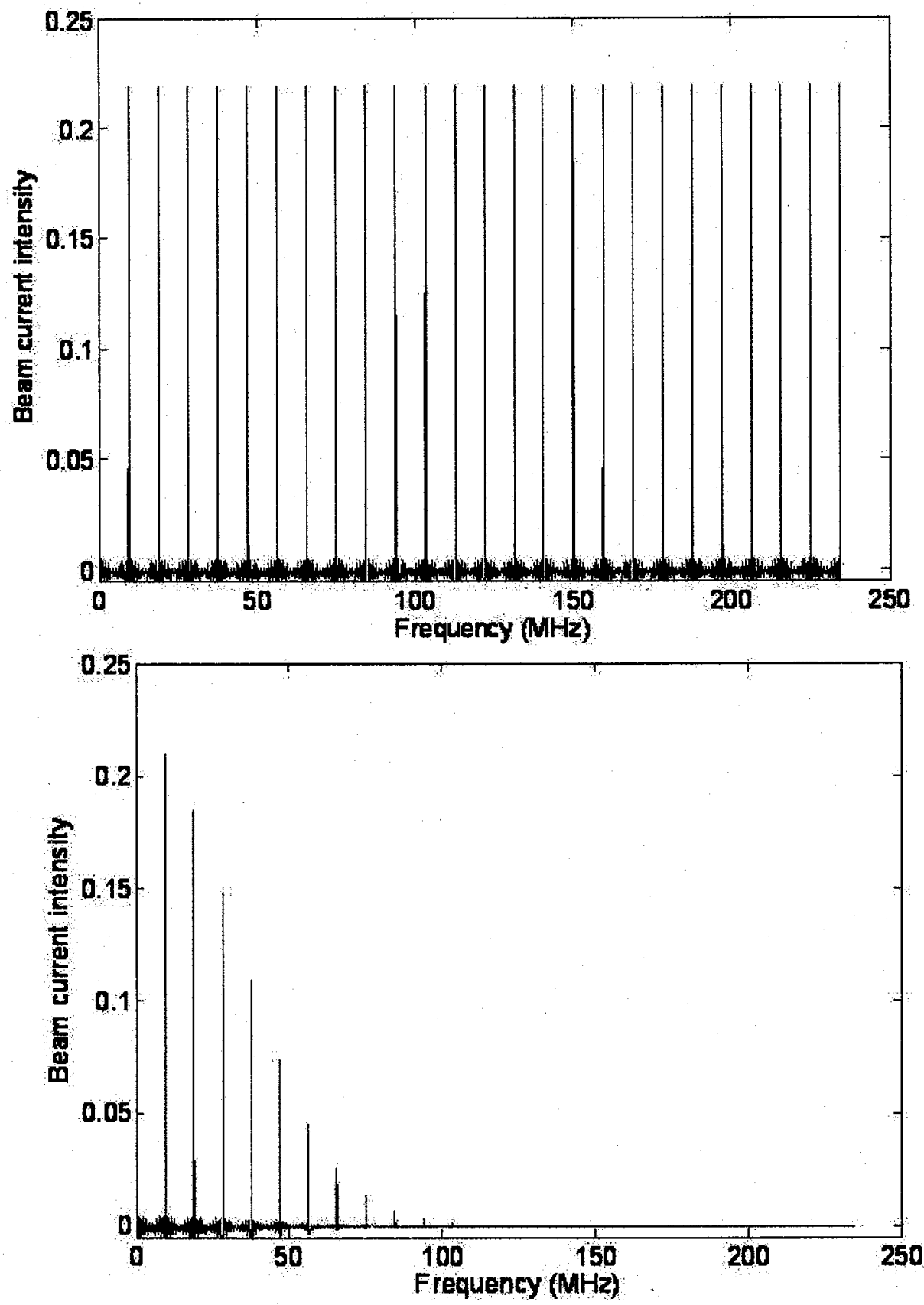

Figure 4: The frequency contents of the beam current: a train of point-charge bunches (top) and a train of gaussian bunches (bottom) 
Table 3: Summary of beam frequencies and cavity frequencies at injection and store

\begin{tabular}{c|c|c|c}
\hline Status & $\gamma$ & $\mathrm{f}_{\text {beam }}(\mathrm{MHz})$ & $\mathrm{f}_{\text {cavity }}(\mathrm{MHz})$ \\
\hline Injection & 25.4 & 56.257 & $\mathrm{f}_{\text {cavity,store }}(56)-\Delta \mathrm{f}_{F D}-\Delta_{\text {detune }}$ \\
& & 168.773 & $\mathrm{f}_{\text {cavity,store }}(168)-\Delta \mathrm{f}_{F D}-\Delta_{\text {detune }}$ \\
\hline Store & 100 & 26.299 & $\mathrm{f}_{\text {cavity,store }}(56)$ \\
& & 168.896 & $\mathrm{f}_{\text {cavity,store }}(168)$ \\
\hline
\end{tabular}

Table 4: Frequency change due to the various dampers and a slow tuner

\begin{tabular}{c|c|c|c}
\hline & $\Delta \mathrm{f}_{F D}$ & $\Delta \mathrm{f}_{H O M}$ & $\Delta_{\text {detune }}$ \\
\hline $56 \mathrm{MHz}$ & decrease by $0.06 \%$ & increased by $0.01 \%$ per a damper & $17 \mathrm{kHz}$ per $1 \mathrm{~mm}$ \\
$168 \mathrm{MHz}$ & decreased by $0.4 \%$ & increased by $0.01 \%$ per a damper & $56 \mathrm{kHz}$ per $1 \mathrm{~mm}$ \\
\hline
\end{tabular}

go through the cavity's resonance and the cavity frequency will be detuned by around $10 \mathrm{kHz}$ from the beam line by a slow tuner. However, at store, the fundamental mode damper will be withdrawn and the detuned cavity frequency will be restored so that the resulting cavity frequency becomes around $500 \mathrm{~Hz}$ offset relative to the beam frequency.

Table 3 summarizes the change of beam frequencies and cavity frequencies at injection and store where $\Delta \mathrm{f}_{F D}$ and $\Delta_{\text {detune }}$ refer to the frequency change due to the fundamental damper insertion and the frequency detune due to the slow tuner, respectively. The fundamental mode and the first HOM of the cavity are considered since these are the modes which give the most of the HOM power as will be discussed later. At store, the cavity frequency will be adjusted so that the frequency difference between the cavity and the beam at the fundamental mode is around $500 \mathrm{~Hz}$. Some parameters defined in Table 3 can be found in Table 4 . In Table 4 , the effect of the fundamental damper is estimated based on the measurement results, which is confirmed in the MWS simulation. As for the HOM damper effect, the MWS simulation results are used for the estimation since the HOM damper has not been tested yet (In fact, one can rely on the simulation results for this estimation since the previous study on the fundamental damper shows very good agreement between the measurement and the simulation [7]). The cavity has been optimized based on the Superfish simulation [4] including the corrugations for eliminating multipacting. The final geometry of the $\mathrm{Nb}$ cavity is shown in Fig. 5. The exact cavity frequencies of the fundamental mode and thet first HOM are obtained from the final

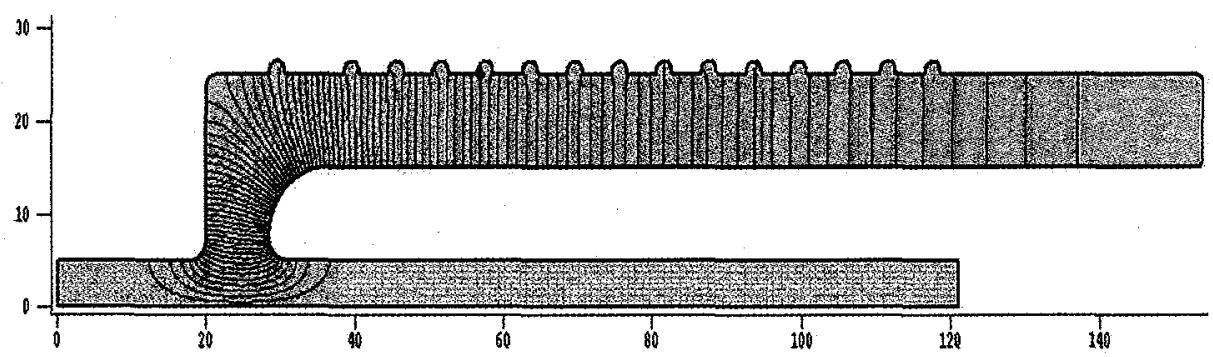

Figure 5: The final cavity geometry simulated in Superfish (Courtesy X. Chang) 
Table 5: Final frequencies at injection and store

\begin{tabular}{c|c|c|c|c}
\hline Modes & \multicolumn{2}{|c}{ Beam frequency $(\mathrm{MHz})$} & \multicolumn{2}{c}{ Cavity frequency $(\mathrm{MHz})$} \\
\hline & injection & store & injection & store \\
\hline $56 \mathrm{MHz}$ & 56.257 & 56.299 & 56.255 & 56.299 \\
$168 \mathrm{MHz}$ & 168.773 & 168.896 & 166.786 & 167.489 \\
\hline
\end{tabular}

Table 6: Summary of the calculated HOM power (For the $56 \mathrm{MHz}$ (fundamental mode), $-45 \mathrm{~dB}$ rejection is considered.)

\begin{tabular}{c|c|c|c|c}
\hline Frequency $(\mathrm{MHz})$ & $\mathrm{Q}_{L}$ & $\begin{array}{c}\mathrm{P}_{\text {res }}(\mathrm{mW}) \\
\text { all resonant case }\end{array}$ & $\begin{array}{c}\mathrm{P}_{\text {inj }}(\mathrm{mW}) \\
\text { at injection }\end{array}$ & $\begin{array}{c}\mathrm{P}_{\text {store }}(\mathrm{mW}) \\
\text { at store }\end{array}$ \\
\hline 56 & 4255 & 1980 & 1740 & 1980 \\
168 & 1818 & 32160 & 40.4 & 88.5 \\
276 & 1614 & 2.05 & 1.46 & 2.05 \\
378 & 1780 & 0.56 & 0.56 & 0.56 \\
475 & 2040 & 0.27 & 0.27 & 0.27 \\
574 & 2580 & 0.11 & 0.11 & 0.11 \\
680 & 2520 & 0.05 & 0.05 & 0.05 \\
789 & 3800 & 0.015 & 0.015 & 0.015 \\
899 & 50400 & 0.0007 & 0.0007 & 0.0007 \\
1008 & 5726 & 0.0064 & 0.0063 & 0.0063 \\
1112 & 4900 & 0.02 & 0.02 & 0.02 \\
1137 & 11060 & 0.005 & 0.005 & 0.005 \\
\hline Total & & $34143 \mathrm{~mW}$ & $1783 \mathrm{~mW}$ & $2072 \mathrm{~mW}$ \\
\hline
\end{tabular}

geometry. The calculation of HOM power is to be done in the following manner: First, the resonant case in which all cavity HOM frequencies overlap with the beam frequencies is considered. Second, the power at injection will be dealt. Lastly, the HOM power at store will be considered.

For estimating the HOM power, the highest HOM power constructs the worst case scenario. Knowing the worst case scenario enables to be equipped with the conservative design of the structure and set up the bottom line of the beam stability. Table 5 shows the resulting frequencies at different stages found from Table 3 . Using the frequencies in Table 5, the HOM power is estimated. Table 6 is a summary for the estimated HOM power when the beam is a train of gaussian bunches. As indicated in Table 6 , the fundamental mode, $56 \mathrm{MHz}$, is considered to be rejected by $-45 \mathrm{~dB}$ from the HOM high pass filter. From the all resonance case, the total power is around $34 \mathrm{~W}$ out of which most of the HOM power is due to the fundamental mode $(56 \mathrm{MHz})$ and the first HOM $(168 \mathrm{MHz})$. It is worthwhile to note that the HOMs whose frequency is greater than $276 \mathrm{MHz}$ have almost negligible HOM power, less than $3 \mathrm{~mW}$ even at the resonance case. Therefore, it indicates that knowing the exact HOM frequencies in order to analyze the amount of HOM power is not necessary. For estimating the HOM power at injection and store, the exact frequencies of the HOMs whose frequency is above $276 \mathrm{MHz}$ are not used. All are assumed to be on resonant, which gives the most conservative estimate. However, the $56 \mathrm{MHz}$ and the $168 \mathrm{MHz}$ frequencies at injection are taken from the estimated frequency 
change due to the presence of the fundamental damper and the HOM damper with $10 \mathrm{kHz}$ cavity detuning. The total power at injection is less than $2 \mathrm{~W}$. At store, the $56 \mathrm{MHz}$ and the $168 \mathrm{MHz}$ come to close to the beam frequency. At this case, the cavity frequency is $500 \mathrm{~Hz}$ smaller than the beam frequency after the cavity is re-detuned by $10 \mathrm{kHz}$. The total HOM power at store is around $2 \mathrm{~W}$. Therefore, the estimated amount of HOM power is a few Watts $(2 \mathrm{~W})$ for both at injection and at store. This enables to simplify the cryogenic design of the cavity.

\section{Summary}

The HOM frequencies and Q factors are analyzed using the available codes: MWS and Omega3P. The Omega3P is used at the first time and compared to the MWS results. Those simulation results between the MWS and Omega3P are in reasonable agreement. The HOM power is estimated analytically, based on the fact that the $28 \mathrm{MHz} H O M$ power measurement was in good agreement with the analytic estimation [6]. The HOM power when all HOMs are on resonant to the beam frequencies is around $40 \mathrm{~W}$. Based on the estimated HOM power, one can construct the engineering design of the HOM damper and the cavity. The actual cavity will have at least 2 HOM dampers.

\section{Acknowledgement}

The author would like to thank Xiangyun Chang for providing the Superfish simulation result.

\section{References}

[1] CST Microwave Studio Suite 2008.

[2] Omega3P User's Manual - http://cubit.sandia.gov.

[3] Cubit User's Manual User Manual - https://mpi.slac.stanford.edu:8443/ext/index.php/Omega3P.

[4] Cubit User's Manual User Manual - http://laacg1.lanl.gov/laacg/services/services.

[5] E. M. Choi and H. Hahn, C-A/AP/319, 2008.

[6] N. Abreu and E. M. Choi, C-A/AP/320, 2008.

[7] E. M. Choi and H. Hahn, C-A/AP/314, 2008. 